\title{
Systematic review of anticholinergic risk scales in older adults. Response to letter of the editor
}

\author{
Carlos E. Durán • Majda Azermai • Robert Vander Stichele
}

Received: 14 May 2013 / Accepted: 21 May 2013 / Published online: 6 June 2013

(C) Springer-Verlag Berlin Heidelberg 2013

\section{Dear Editor,}

A uniform drug list for screening the anticholinergic burden in medication lists of the elderly might indeed be an important tool to assure patient safety. There will always be discussions on what to include and what not to include, based on clinical relevance and epidemiological prevalence. Important for the selection is the requirement for a published clinical study probing the relationship between alleged anticholinergic effect and clinical outcomes (or surrogate endpoints) in an empirical observational or explanatory design. It is a requirement similar to the requirement for a pivotal trial or systematic review as evidence for the recommendation to use a specific drug in a specific indication. This collection of empirical evidence on adverse effects has not been performed for all known anticholinergics. The publication of our list in the EJCP has triggered scientists and clinicians to put forward additional active ingredients as potential candidates for the list, either drugs from local markets, or specialized disciplines (pneumology, urology, neurology, etc.). We welcome those suggestions, and invite the research community to subject these drugs to clinical tests capable of providing information on their intrinsic potency in clinical practice.

Adapting a uniform international list to local markets is especially important in countries with less regulated markets, as in some developing countries.

Establishing a uniform drug list of anticholinergics with a simple grading of the intrinsic potency was a first step. We strongly believe that a necessary next step is to determine, for each anticholinergic drug on the list, information on minimal, optimal and maximal dosing in the elderly. It is another prerequisite for building a credible screening tool for anticholinergic burden among polymedicated elderly, that will survive validation efforts in clinical practice [1].

\section{Reference}

1. Kersten H, Molden E, Tolo IK, Skovlund E, Engedal K, Wyller TB (2013) Cognitive effects of reducing anticholinergic drug burden in a frail elderly population: a randomized controlled trial. J Gerontol A Biol Sci Med Sci 68:271-278

\section{E. Durán}

Centro de Biomedicina, Universidad Central del Ecuador,

Quito, Ecuador

M. Azermai $\cdot$ R. Vander Stichele $(\bowtie)$

Heymans Institute of Pharmacology,

Ghent University, Ghent, Belgium

e-mail: robert.vanderstichele@ugent.be 